\title{
The impact of temporal complexity reduction on a $100 \%$ Renewable European Energy System with Hydrogen Infrastructure
}

\author{
Dilara Gulcin Caglayan, ${ }^{1,2 *}$ Heidi Ursula Heinrichs, ${ }^{1}$ Detlef Stolten ${ }^{1,2}$ and Martin Robinius ${ }^{1}$ \\ ${ }^{1}$ Institute of Energy and Climate Research - Electrochemical Process Engineering (IEK-3) \\ Forschungszentrum Jülich GmbH, 52425 Jülich, Germany \\ ${ }^{2}$ RWTH Aachen University, Chair for Fuel Cells, Faculty of Mechanical Engineering, \\ Kackertstraße 9, D-52072 Aachen, Germany
}

(*)d.caglayan@fz-juelich.de

\begin{abstract}
The transition towards a renewable energy system is essential in order to reduce greenhouse gas emissions. The increase in the share of variable renewable energy sources (VRES), which mainly comprise wind and solar energy, necessitates storage technologies by which the intermittency of VRES can be compensated for. Although hydrogen has been envisioned to play a significant role as a promising alternative energy carrier in a future European VRES-based energy concept, the optimal design of this system remains uncertain. In this analysis, a hydrogen infrastructure is posited that would meet the electricity and hydrogen demand for a $100 \%$ renewable energybased European energy system in the context of 2050. The overall system design is optimized by minimizing the total annual cost. Onshore and offshore wind energy, open-field photovoltaics (PV), rooftop PV and hydro energy, as well as biomass, are the technologies employed for electricity generation. The electricity generated is then either transmitted through the electrical grid or converted into hydrogen by means of electrolyzers and then distributed through hydrogen pipelines. Battery, hydrogen vessels and salt caverns are considered as potential storage technologies. In the case of a lull, stored hydrogen can be re-electrified to generate electricity to meet demand during that time period. For each location, eligible technologies are introduced, as well as their maximum capacity and hourly demand profiles, in order to build the optimization model. In addition, a generation time series for VRES has been exogenously derived for the model. The generation profiles of wind energy have been investigated in detail by considering future turbine designs with high spatial resolution. In terms of salt cavern storage, the technical potential for hydrogen storage is defined in the system as the maximum allowable capacity per region. Whether or not a technology is installed in a region, the hourly operation of these technologies, as well as the cost of each technology, are obtained within the optimization results. It is revealed that a 100 percent renewable energy system is feasible and would meet both electricity demand and hydrogen demand in Europe.
\end{abstract}

Keywords: Renewable energy systems, energy systems, hydrogen pipeline, power-to-hydrogen. 


\section{INTRODUCTION}

Approximately $75 \%$ of global greenhouse gas emissions in 2017 comprised carbon dioxide, $90 \%$ of which were the result of fossil fuel combustion [1]. With the goal $80 \%$ to $95 \%$ greenhouse gas emission reduction against 1990 levels by 2050, renewable energy is of high importance to the energy system transition. The increase in the installed capacities of renewables over the last decade can also be interpreted to understand their role and infer the shift towards higher renewable energy shares in the energy mix. The increase in the global renewable energy capacity between 2010 and 2018 is reported as $1127 \mathrm{GW}$, with nearly $75 \%$ of this capacity being constituted by wind and solar technologies [2]. Being environmentally-friendly, globally available and showing decreasing cost, wind and solar energy technologies, as variable renewable energy sources (VRES), have the drawback of intermittency [3]. In order to overcome this obstacle, chemical energy carriers, which are produced at peak generation time periods, have been proposed as a mitigating option. Thus, such a chemical energy carrier can be used to balance the grid or as a feedstock for other processes [4]. Amongst many possible chemical energy carriers, hydrogen is considered to be amongst the most promising owing to its high energy density and carbon-free nature. Hydrogen can be produced directly by splitting water molecules into hydrogen and oxygen, which is called electrolysis. It can also be used in many industries, such as ammonia, steel, iron, polymer production, as well as in petroleum refineries [5]; furthermore, it can serve as fuel in fuel cell-electric vehicles [4]. As a result, different sectors can be coupled by means of hydrogen [6].

Several studies conceptualize a future European energy system based on large shares of renewables without the use of hydrogen as a chemical energy carrier. Nevertheless, many key methodological aspects of these would not significantly change with the addition of hydrogen. For instance, Aboumahboub et al. [7] models both the global and European energy systems assuming $100 \%$ renewable utilization with an objective function of the minimization of the total annual cost. In their study, the method employed to obtain renewable energy time series is not explained, but the optimization results reveal a total European capacity of 6 TW to 6.7 TW, a large proportion of which is from wind energy [7]. Moreover, 10\% of these capacities are made up by solar energy. Siala and Mahfouz [8] performed an analysis of the influence of regionalization of the European energy system. Assuming 2015 as the weather year, and the second weeks of January, April, July and October as the representative time series for that year, they optimized the system [8]. Zappa et al. [9], meanwhile, conceived a 100\% renewable energy system at the European level using 2010 as the weather year. However, the optimization is performed by using monthly load duration curves, which is then followed by hourly simulations for the unit commitment and economic dispatch model [9]. However, these hourly simulations only involve the first week of each month with a solution time limitation of 2 hours [9]. All in all, it must be noted that despite the interesting analyses, none of the previously mentioned studies take hydrogen into account in the energy system design. 
European analyses conducted by Bussar et al. [10-12] employ 21 regions in order to design the system in the context of 2050. The approach used to obtain the hourly time series is not explicitly indicated in any of the studies mentioned. A wind-to-solar ratio of 40:60 is assumed in all of them. Although hydrogen storage and conversion technologies are taken into account, the transmission of hydrogen is not modeled [10-12]. Grid versus storage is investigated by Steinke et al. [13] for a 100\% renewable energy system, with a wind-to-solar ratio of 65:35. Although the use of 8-year weather data is acknowledged, the method used for estimating the time series remains unclear [13]. A major drawback of this analysis is the consideration of single storage technology for each model run; in other words, the combination of hydropower, batteries and hydrogen storage is not addressed [13]. A future energy system design and its evolution are investigated by Schlott et al. [14] in the context of 2100. In this analysis, the years between 1970 and 2100 are grouped into 4 periods with 6-8 yearly model runs and 3-hourly temporal resolution [14].

Although the aforementioned studies consider hydrogen to some degree in the design of the energy system, none of them fully address all of the necessary characteristics of a future system. Firstly, consideration of future-oriented wind and solar technologies is lacking, although the analyses are conducted in a future-looking context. Secondly, the temporal resolution of these studies does not cover the entire year with hourly time series. Rather, either three-hourly time series or representative periods are employed. Finally, although hydrogen is considered a commodity, hydrogen transmission between regions is not modeled in any of these studies.

Both the design of future energy systems and the utilization of hydrogen as an energy carrier have been investigated by several studies in the literature [15-17]. Designing a hydrogen infrastructure has mostly been performed at a national level, generally assuming the country of focus to be an island without any interactions with neighboring countries [15]. In contrast, when imports and exports are taken into account with a detailed electrical grid, hydrogen infrastructure is not usually incorporated into the analysis [18]. This is generally observed in power flow simulations with a multinational spatial system boundary. Alongside consideration of the hydrogen infrastructure, three main points should be addressed in design analyses. First, a detailed spatial and temporal resolution of the models is vital to accounting for the spatial variability of VRES technologies. Secondly, as a future context is assumed, the considered technologies should also reflect future characteristics, such as larger wind turbines and improved efficiencies of components, e.g., electrolyzers or fuel cells. Finally, energy systems that include VRES sources and hydrogen as commodity technologies are likely to also require seasonal storage technologies. Therefore, consideration of geological underground storage, including a founded analysis of their storage potentials is crucial, as it has been proven that salt caverns are the most economical seasonal storage technology [19]. Up until now, all of these points have not yet been addressed in the literature.

In this analysis, a European hydrogen infrastructure to supply electricity and hydrogen based on $100 \%$ renewable energy sources is designed in the context of 2050 . It utilizes hydrogen as a 
chemical energy carrier and considers different renewable energy sources alongside the electrical grid. The potentials and generation profiles of wind and solar technologies are obtained by drawing future designs derived from a detailed pre-analysis based on the work of Ryberg et al. [20] and Caglayan et al. [21]. In addition, the maximum capacities of salt caverns available within each region are also investigated by means of a dedicated pre-analysis based on the methodology suggested by Caglayan et al. [22]. The optimizer determines whether or not a technology should be installed, as well as the capacity to be installed, with an objective function of minimizing the system's total annual cost (TAC). Additionally, the hourly operation of all technologies is obtained as a result of the analysis.

\section{METHODOLOGY}

In order to perform the analysis, an open-source optimization framework called the Framework for Integrated Energy System Assessment (FINE) [23] is employed, the formulation and constraints of which are explained in detail by Welder et al. [15]. The objective function of the optimization is to minimize the TAC of the system. The framework can be used to develop optimization models with different spatial and temporal resolutions, but in this analysis the regional scope is defined as Europe. The region of interest is divided into 96 smaller regions following the regional boundaries suggested by the E-Highway study [24] (see Figure 1). The temporal resolution of the problem has been altered to observe the impact of the time series aggregation with a large regional scope. Nevertheless, without time series aggregation, the model consists of hourly data for one year, which is taken as 2050 in this study.

Figure 1 illustrates the system configuration employed in this analysis, in which two commodities are considered: hydrogen and electricity. Therefore, the blue and green boxes represent electricity and hydrogen and the technologies shown for these areas work with the corresponding commodity. Given hourly demand and generation, conversion, transmission and storage technologies for electricity and hydrogen for each defined region, the overall system design and operation of the technologies can be obtained as a result of the optimization. Each region contains a specific technology portfolio and expansion options and regions can interact by using either the electricity grid or hydrogen grid. 


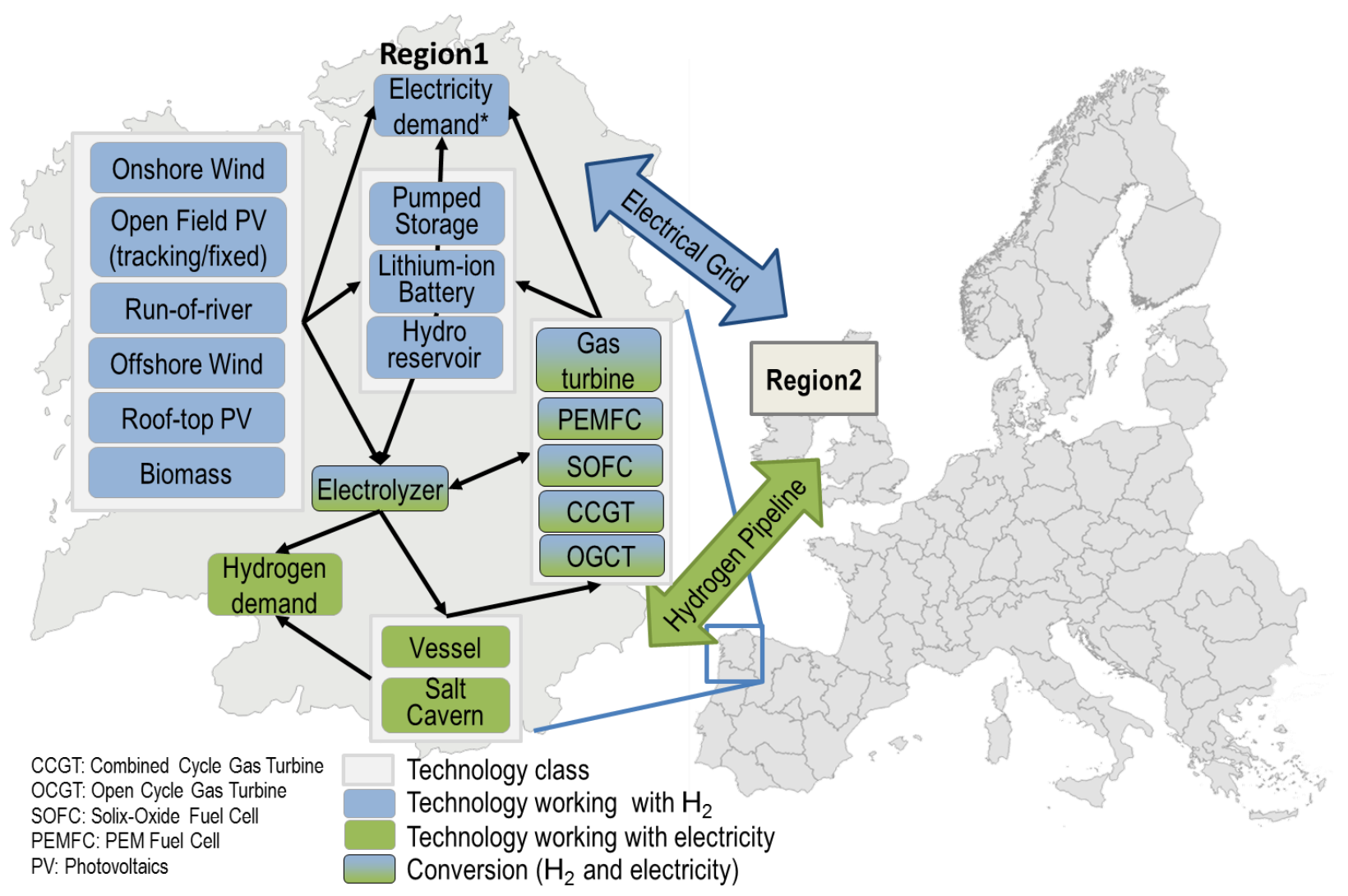

Figure 1. Technologies and the selected system boundaries employed in this analysis.

\subsection{Hydrogen and Electricity Demand}

Electricity demand is estimated by using the methodology suggested in the E-Highway study [24]. This approach provides electricity demand by also including the large share of electrified heat demand and transport demand for battery-electric and plug-in hybrid vehicles. In this work, transport demand is excluded from the electricity demand, as the methodology used in the estimation of electric vehicle demand differs from that of fuel cell-electric vehicles. Referring to the previous analyses $[6,17,25]$, hydrogen demand for fuel cell-electric vehicles will be taken into account instead of that of electric vehicles.

In order to obtain the yearly demand for hydrogen, the driving behavior of existing vehicles is transposed to fuel cell-electric vehicles. Annual driving distance and the number of passenger vehicles for each country in the context of 2050 is obtained from the E-Highway "100\% RES" scenario [24], as it provides consistent data for the region of interest. Following this, overall demand is distributed across each country with the population density distribution. Temporal resolution is obtained by projecting yearly demand onto an hourly profile of a fueling station.

\subsection{Generation Technologies}

In order to maintain the consistency amongst renewable technologies, historical data for 2015 are used, both as the weather year and also for the electricity demand. The operation time series for 
onshore and offshore wind energy, open-field and rooftop photovoltaic (PV) are calculated using the Modern-Era Retrospective analysis for Research and Applications (MERRA-2) dataset [26]. Run-of-river operation time series are obtained from Syranidis et al. [27]. Finally, biomass capacity defined by the E-Highway " $100 \%$ RES" Scenario [24] is introduced into the model. The economic parameters for PV, hydropower and biomass technologies are assumed in accordance with the reference scenario by Arantegui et al. [28]. Moreover, these parameters are assumed to be constant for all regions. However, for onshore and offshore wind energy, cost variation with respect to turbine location and size is estimated by the cost models [21,29].

Figure 2 shows the full load hours (FLH) of onshore and offshore wind energy installations across each region, as well as their specific cost as a demonstration for the spatial resolution of this analysis. It must be noted that higher FLH are due to the consideration of future turbine designs, which has not been addressed in detail in the energy system design. Additionally, the spatial resolution shown in Figure 2 represents a strength of the approach presented here. The applied approach allows similar results to be obtained for every technology. In particular, the specific costs of wind turbines vary significantly, as the cost is always site-specific. For instance, although high full load hours are observed around Italy, the specific costs of the wind turbines are 2-3 times higher than those in the near-shore North Sea region. It must be noted that the average specific cost per region is calculated and defined in the model as a simplification, as optimization problems can easily become intractable.
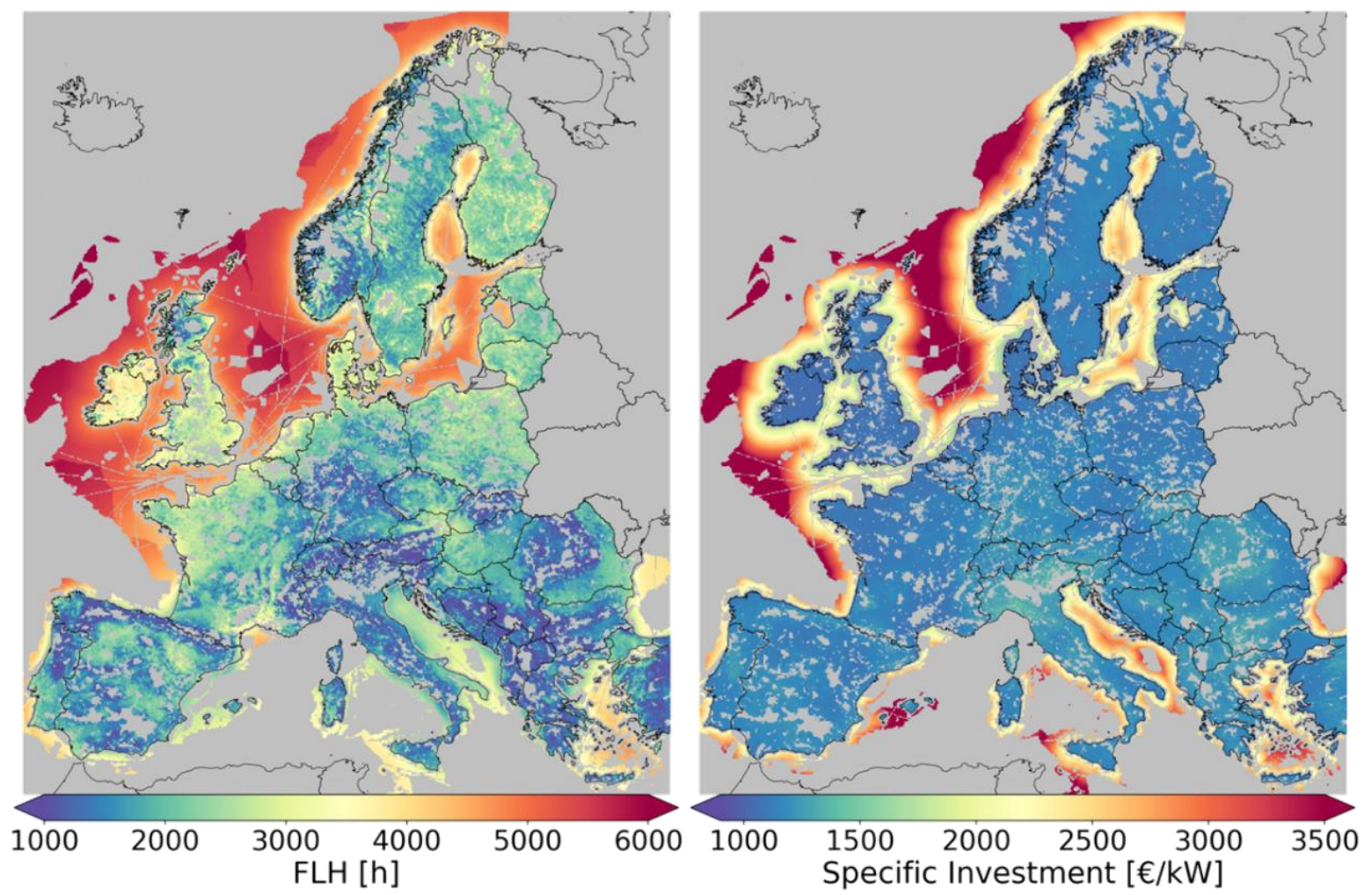

Figure 2. Distribution of FLH (left) and specific cost (right) of onshore and offshore wind energy. 


\subsection{Conversion Technologies}

Six conversion technologies are included for conversion between electricity and hydrogen in this analysis: Polymer electrolyte membrane (PEM) electrolyzers, polymer electrolyte membrane (PEM) fuel cells, solid oxide fuel cells (SOFCs), hydrogen combine cycle gas turbines (CCGTs), hydrogen open cycle gas turbines (OCGTs) and hydrogen gas engines. The production of hydrogen is conducted by means of electrolyzers, which are electrochemical devices for splitting water into hydrogen and oxygen using electricity. Alkaline electrolysis, polymer electrolyte membrane (PEM) electrolysis and solid oxide electrolysis are considered the electrolyzer technologies with different technology readiness levels. Being still in the development phase, solid oxide electrolyzers operate at high temperatures (around 600-1000 ${ }^{\circ} \mathrm{C}$ ), causing thermal stress on the ceramics [4,30]. Both alkaline electrolyzers and PEM electrolyzers require high hydrogen purity and a fast response time of nearly 20 minutes [30,31]. While alkaline electrolyzers consist of hazardous electrolyzers, precious metals are required for PEM electrolyzers [30]. Taking into account the aforementioned aspects of these technologies, it was decided to only include PEM electrolyzers in the energy system design. In terms of the reelectrification of hydrogen, several different options are included, in accordance with the technologies assessed by Welder et al. [32]. For this purpose, the optimizer is used to decide which technology to use in the re-electrification pathways of hydrogen amongst the five technologies mentioned previously.

\subsection{Storage Technologies}

There are several technologies that can be used for the storage of hydrogen and electricity. In terms of electricity storage, pumped hydro storage and hydro reservoirs are taken into account without allowing for capacity expansion following the similar methodology applied in the literature $[18,33]$. The determination of the potential locations for these technologies and also the inflow time series requires a more detailed analysis with appropriate data, which is also beyond the scope of this work. Therefore, the capacities of these technologies are kept constant at the values reported for 2015, as well as the water inflow time series. These data are derived from the work of Syranidis et al. [27]. In addition, lithium-ion batteries are considered for electricity storage.

Gas vessels and salt caverns are considered as hydrogen storage technologies. In particular, salt caverns are the most promising seasonal storage technology due to their high storage capacities and relatively lower costs, as is also stated by Crotogino et al. [19]. Consideration of salt caverns should be emphasized, as they have not been investigated on a European scale in an energy system model. Including thin-bedded and salt domes, an eligibility assessment is performed on European salt deposits. Following this, the technical potential is determined by distributing salt caverns and calculating cavern potential based on the thermo-dynamic properties of hydrogen at each location. Finally, the maximum technical potential in terms of capacity is extracted from the results of the aforementioned assessment and integrated into the model. In order to consider the 
seasonal storage of hydrogen as a possibility in the regions that do not have salt cavern potential, vessels are taken into account as a second storage technology.

\subsection{Transmission Technologies}

Two transmission technologies are considered for electricity transmission between regions: highvoltage alternating-current (HVAC) and high-voltage direct-current (HVDC) lines. Consideration of HVAC and HVDC connections in the context of 2050 is not possible due to the lack of data. Electricity transmission is taken into account with the grid enhancements as per the "Ten Year Network Development Plan (TYNDP)" [34], the methodology of which is explained in Syranidis et al. [27]. However, the electrical grid is exogenously fixed without investment costs, and further grid enhancements beyond the TYNDP are prohibited.

In terms of hydrogen transportation between regions, only pipelines are considered transmission technologies, as they are found to be the cheapest transportation method for large-scale transportation across long distances [35]. Only newly built hydrogen pipelines are considered; since the technology is concluded to have a business case [4]. The routing of these pipelines is conducted by calculating the shortest path between regions by using a candidate grid consisting of existing natural gas pipelines [36], railways, highways and primary roads. ${ }^{1}$ The routing and estimation of distances is only performed between neighboring regions.

\section{RESULTS}

This section consists of the results obtained by using the proposed methodology to design the energy system, and is divided into two discussions. The first part considers the reference system design by means of capacity distribution, as well as the optimal operation of the system components. The second part provides insight into the impact of time series aggregation on the energy system design, and how the different number of typical periods affects the overall system in terms of cost and design. These results are compared against the reference system design, which is obtained without the application of time series aggregation.

\subsection{0\% Renewable European Energy System Design}

Figure 3 illustrates the system design obtained without time series aggregation. In order to conduct a succinct discussion, other technologies such as batteries or electrolyzers are not included in the plot. The optimal system design consists of $737 \mathrm{GW}$ of onshore, $125 \mathrm{GW}$ of offshore and $844 \mathrm{GW}$ of open-field PV without tracking. Additionally, $33 \mathrm{GW}$ of biomass CHP plants are installed. Amongst the generation technologies, it can be seen that rooftop PV and open-field PV with tracking are not installed anywhere. This is mainly because of their high investment costs compared to open-field PV without tracking. It must be noted that generation with these two PV technologies is not significantly better than open-field PV without tracking;

\footnotetext{
${ }^{1}$ The data for railways, highways and primary roads are obtained by using the "prior dataset" of
} GLAES [37]. 
nevertheless, the investment cost of PV panels with tracking systems is nearly $36 \%$ higher than that without tracking system [28]. In case of rooftop PV, the cost deviates by nearly $70 \%$ (rooftop PV has a higher investment cost). Although slight increases in the generation can be achieved with these technologies, they are not found to be necessary in the system. In other words, slightly higher generation in the early morning and late evening obtained by open-field PV with tracking does not compensate its relatively higher investment cost.

Differing than the results presented by Schlott et al. [14], Europe is not divided into two in terms of wind and PV locations. In fact, the installation of PV can be seen in several regions in the north (cf. central United Kingdom and northern Poland), despite their comparably low generation potential. Nevertheless, instead of a clear differentiation of wind and PV technologies, hybrid systems are found to provide smoother generation with fewer fluctuations. Therefore, countries such as Ireland, the United Kingdom and Poland utilize the combination of onshore and offshore wind energies, as well as PV technologies. Nevertheless, the preference for open-field PV without tracking in southern Europe is also evident.

Looking at the pipeline capacities reveal that cheap electricity generation locations in Ireland and the United Kingdom could provide large amounts of hydrogen for continental Europe. Furthermore, hydrogen transport from the Nordic countries is also apparent with lower pipeline capacities. It can be seen that pipeline capacities around the regions with salt cavern availability is higher because of the availability of cheap hydrogen storage. An example for this large pipeline capacity and the availability of salt cavern is the connection between northwestern Spain and the southeastern France. The region in France imports hydrogen from both Spanish regions via large pipelines. A total utilization of $187 \mathrm{TWh}$ of salt caverns is seen in the overall system, the largest portions of these capacity deployments being observed in the Netherlands, northwestern Germany, regions in the United Kingdom around Manchester and Liverpool and in the central France region neighboring Paris. In addition to the salt caverns, the deployment of 1 TWh of lithium-ion battery storage and $0.7 \mathrm{TWh}$ of vessels are observed in the system. A detailed capacity distribution can be found in the appendix. 


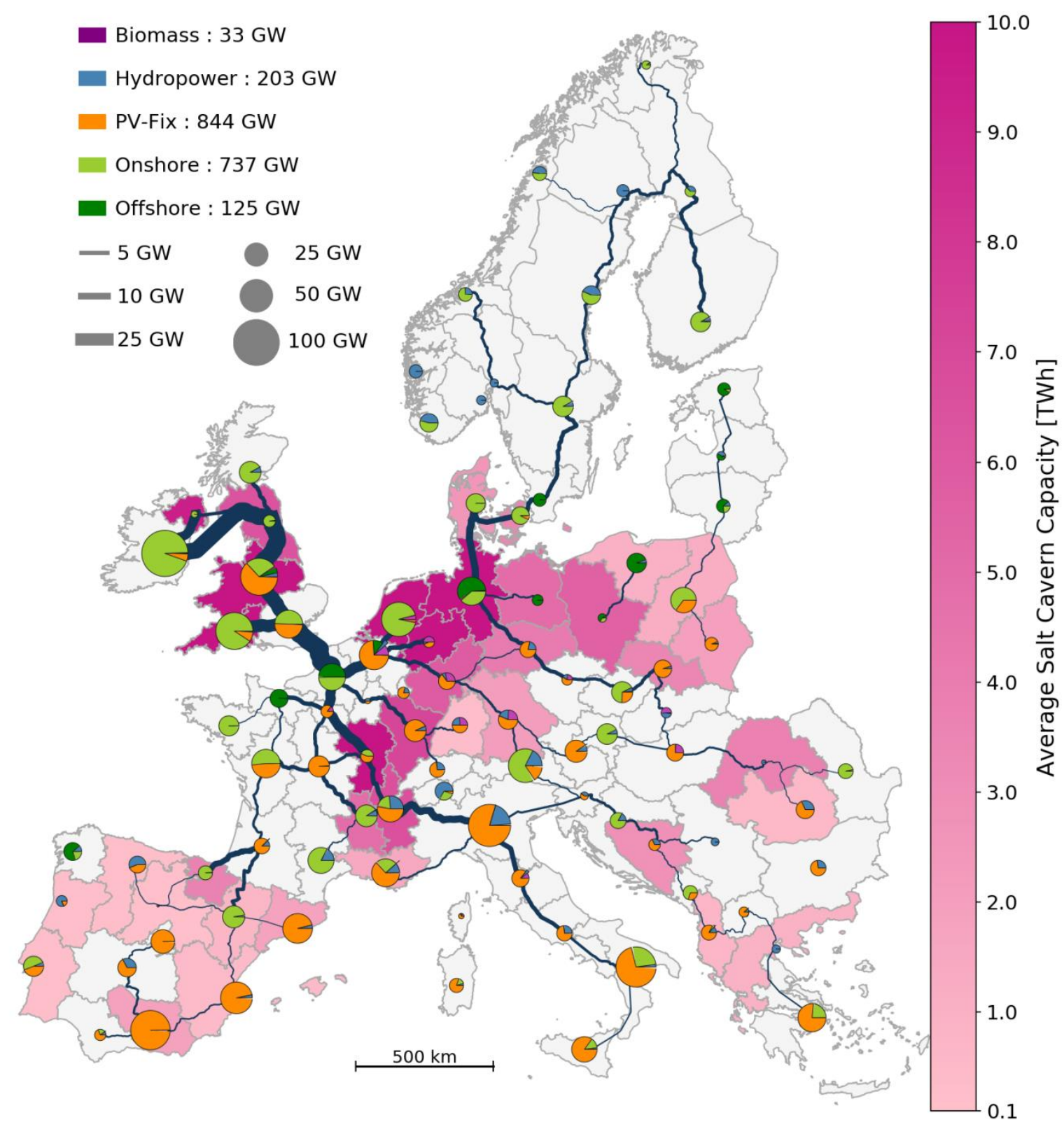

Figure 3. Capacity distribution of VRES technologies, salt caverns and hydrogen pipeline.

Analysis of the optimal capacity distribution reveals that neither open-field PV with tracking nor rooftop PV systems are preferential in the design. This is because of the higher investment cost of these technologies, which prevents them from being cost-competitive against open-field PV without tracking systems. For instance, the investment costs of rooftop PV and open-field PV with tracking systems are nearly 1.7 and 1.4 times higher, respectively, than that of open-field PV without tracking. Moreover, the operation and maintenance costs of these technologies also differ. Nevertheless, comparison by means of only economic parameters is not sufficient, as the individual technologies have different generation time series. Therefore, LCOE can be used to 
understand why previously mentioned technologies are not chosen by the optimizer. Comparison of LCOE values in each region and technology reveals that open-field PV without tracking has the lowest LCOE for all the regions. When the LCOE values of open-field PV with and without tracking systems are analyzed, it can be seen that the discrepancy between the values is more pronounced in the northern European regions. However, a minimum difference of $0.3 €_{\mathrm{ct}} \mathrm{kWh}^{-1}$ is observed in the southern European regions. In the case of rooftop PV, the LCOE values differ by at least $2.6 €_{\mathrm{ct}} \mathrm{kWh}^{-1}$, meaning that it is almost 1.8 to 2.2 times higher than that of open-field PV without tracking. Although rooftop PV in particular is already installed in many regions, the presented model is not able to capture the medium-level transmission and distribution grid. Therefore, the grid bottlenecks and the requirements for rooftop PV are not fully covered within the existing methodology.

The capacity values provided by the E-Highway study ("100\% RES") scenario includes $760 \mathrm{GW}$ onshore, $115 \mathrm{GW}$ offshore and $662 \mathrm{GW}$ PV as VRES technologies besides the $184 \mathrm{GW}$ of biomass CHP plants and $297 \mathrm{GW}$ of hydropower [24]. Comparison of the capacities shows that the results are very similar for wind energy, yet the capacity estimated for open-field PV in this work is nearly $180 \mathrm{GW}$ higher than what is reported in the E-Highway study [24]. This is due to the elimination of $73 \mathrm{GW}$ of fossil fuel plants, half of which are installed in France, Italy and Spain. In the present work, the fossil fuel is substituted by the open-field PV technology, especially in the afore-mentioned countries, which causes this $180 \mathrm{GW}$ discrepancy in the results.

An exemplary operation time series is shown in Figure 4 for the region that includes London as a high demand center. It must be noted that the operation time series can be obtained for any region and any time period between January $1^{\text {st }}$ and December $31^{\text {st }}$. However, in this specific example, the second half of April is illustrated for both hydrogen and electricity flows. The negative flow mean that the commodity is consumed in or transported from the region, whereas the positive values indicate the generation/production of the respective commodities. Focusing on the electricity flow reveals that the region largely relies on electricity imports. In addition, the utilization of onshore wind energy and open-field PV without tracking can also be seen. Unlike what is reported by Schlott et al. [14], solar energy is utilized in the United Kingdom in spite of its relatively low generation potential. At certain time periods, when the demand cannot be supplied by imports, onshore wind energy and open-field PV, the utilization of lithium-ion batteries, as well as the re-electrification of hydrogen, is evident. Furthermore, when the generation is higher than the demand, charging lithium-ion batteries and the use of electricity in the electrolyzers is also to be seen. Although it is not especially clear, the curtailment of energy on April $18^{\text {th }}$ is apparent in the time periods when the generation is apparently larger than that of the demand and energy that can be utilized in the other technologies. Finally, electricity is occasionally exported to the neighboring regions, but the amount is significantly smaller than in the imports. 
Analysis of the hydrogen flow shows that hydrogen demand in this region is mainly supplied by imports via hydrogen pipeline. Nevertheless, the utilization of the electrolyzers can also be seen in the figure. In spite of the relatively small electrolyzer capacity, some portion of the demand is still supplied by local hydrogen production. Salt caverns are not available in this region; nevertheless, a small vessel storage capacity is deployed. Although it is not clearly visible, hydrogen discharge at some periods can be seen, especially at the end of April $23^{\text {rd }}$. Finally, the utilization of re-electrification technologies can be seen in the lower figure too. Note that the lower and upper figures have different scales of the y-axes.

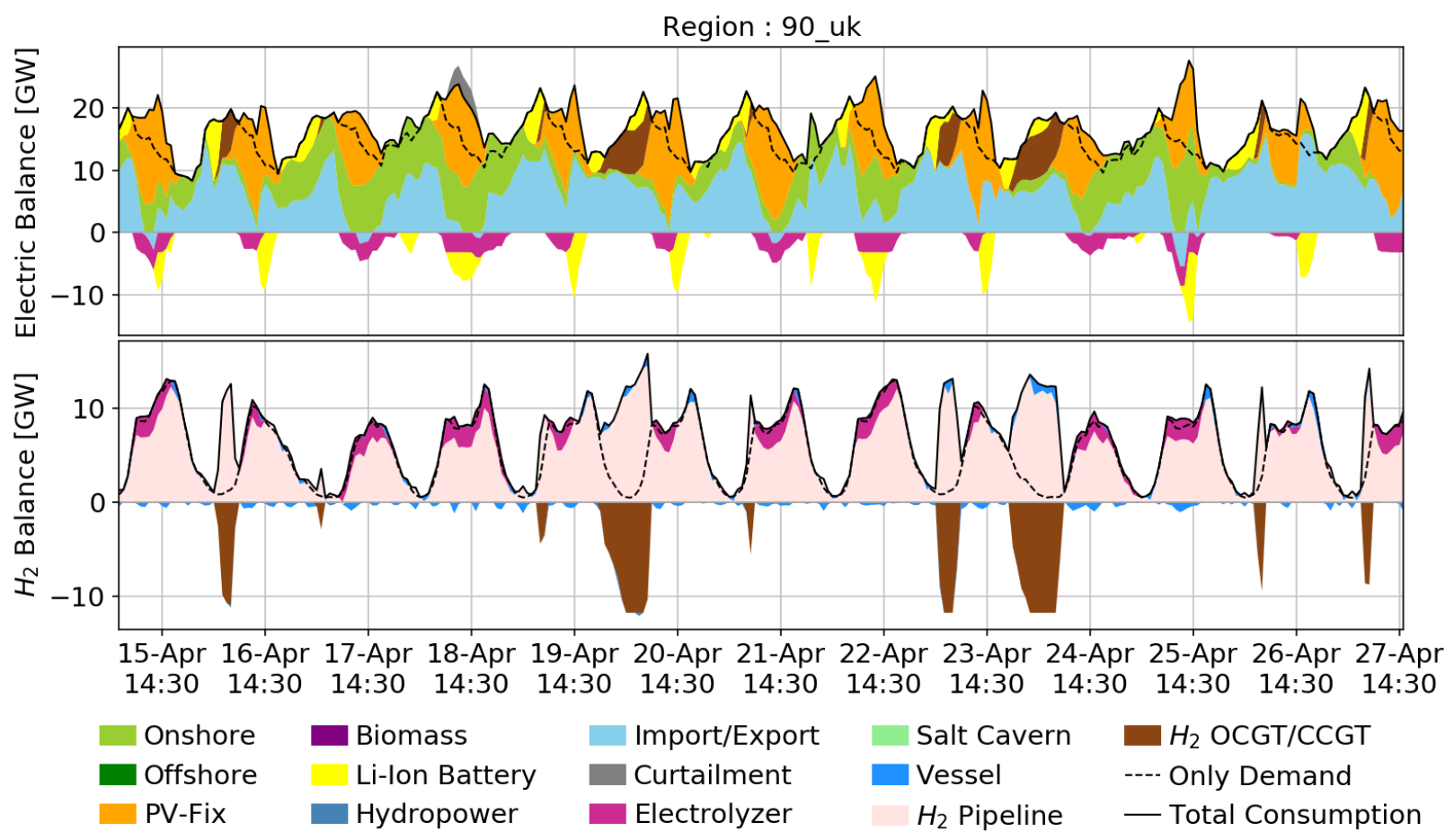

Figure 4. Exemplary operation of individual technologies for the region covering London (upper) electricity, (lower) hydrogen.

\subsection{Impact of Time Series Aggregation on the Design of Energy Systems}

This analysis is conducted by using a different number of typical days to represent the overall energy system design. For representation, 5, 10, 15, 20, 30, 45, 60 and 90 typical days are used. The maximum number of typical days is limited to 90 because of computational memory limitations. The results without time series aggregation are obtained by using a workstation (different than the dedicated computer); as a result, the solution time of the problem is not comparable against other calculations. The solution time for the optimization model is affected by the other processes in the computer; therefore, a dedicated computer is used for model runs to eliminate this impact as much as possible. The computer used for this analysis has 32 GB RAM with Intel Core i7-6700 processors with a $3.40 \mathrm{GHz}$ frequency and 8 threads. The workstation has 500 GB RAM with 2 Intel Xeon Platinum 8176 processors with $2.10 \mathrm{GHz}$ frequency and 112 
threads. As was mentioned earlier, other jobs/processes running in parallel affect the solution time; therefore, the workstation is not used throughout the analysis in order to not occupy the 112 threads. It must be noted that the variation of the solution time is only provided to illustrate the model's complexity and the time elapsed for the analysis rather than to draw conclusions based on these biased values. The numbers might vary significantly for the same problem when another computational setup is used.

The change of solution time for different numbers of typical days is illustrated in Figure 5. It also includes the solution time of the model run without time series aggregation to provide an insight, yet it is not directly comparable because of the different computational setup used for that model run. Unsurprisingly, the solution time increases with higher numbers of typical days. While the solution time is approximately 1.5 hours for 15 typical days, the use of 20 typical days yields 2.5 hours. An unexpected solution time for 30 typical days is obtained, which does not follow the exponential trend seen in the figure. The solution time for this model run is supposed to be between 2.5 and 4.5 hours (values of 20 and 45 typical days, respectively), yet the time elapsed is nearly 8.5 hours. This irregularity might be caused by the other processes (unstoppable tasks undertaken by Windows) running in parallel with this model run. All in all, an exponential increase in the solution time with respect to the number of typical days is apparent.

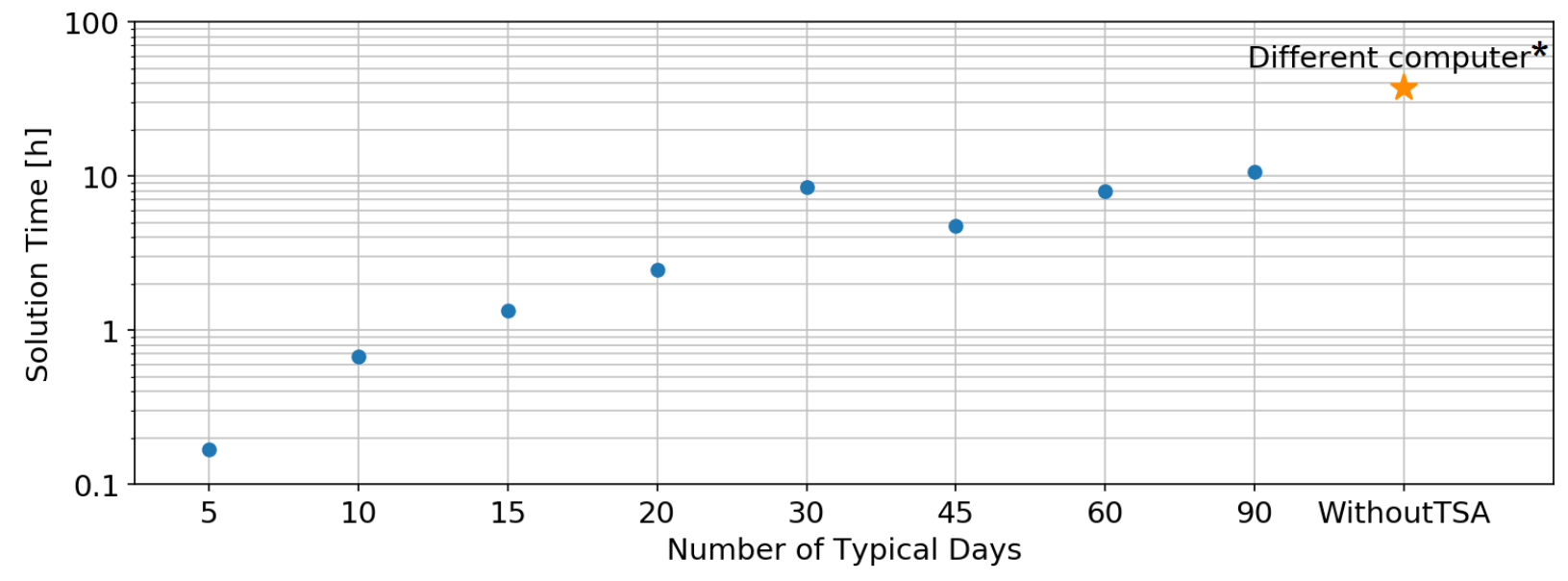

Figure 5. Variation in the solution time by number of typical days (*Calculations for the case without time series aggregation was performed on a different computer).

The time series data defined exogenously in the energy system designs consists of nearly 10 different technologies for 96 regions, all of which have different characteristics. Even the time series of wind energy in the United Kingdom and southern Italy do not have the same profiles, let alone the differences in the technologies. Therefore, the use of a few typical days might not be sufficient to represent an entire year for a large regional context such as Europe. However, as shown previously, a higher number of typical days increases the solution time. Thus, the role of time series aggregation in the complex systems is evident. Use of this tool varies the input time series of the optimization model when the number of typical days is altered; hence, the system 
design might change. In order to investigate this, the cost breakdown of the overall system with respect to different numbers of typical days is shown in Figure 6.

When values below 30 typical days are used, the total annual cost is underestimated. This underestimation is more pronounced, especially between 5 and 10 typical days with a value of $15 \%$. A large variation in the shares of VRES technologies is apparent for these model runs. Moreover, shares of electrolyzer, biomass, salt caverns and hydrogen re-electrification technologies are lower than in the reference scenario. In fact, biomass and hydrogen reelectrification is almost not utilized in these model runs, despite their relatively larger share in the reference scenario. It can be clearly seen that the system cannot be represented by too few typical days, which causes the use of very similar normalized profiles for all technologies. The scaling of these, normalized by the optimal capacities, results in a synchronous time series of source and sink technologies, which eliminates the requirement for the storage and conversion technologies. Therefore, the optimal capacities for these technologies are underestimated when the typical days are not sufficient to represent the system. Nevertheless, there is no rule of thumb to determine the most appropriate number of typical days, as it is a strong function of the fluctuations in the time series, as well as differences in the characteristics of individual technologies. All in all, in spite of the variations between 5 and 30 typical days, variations in the cost of components are insignificant above 30 typical days in this case.

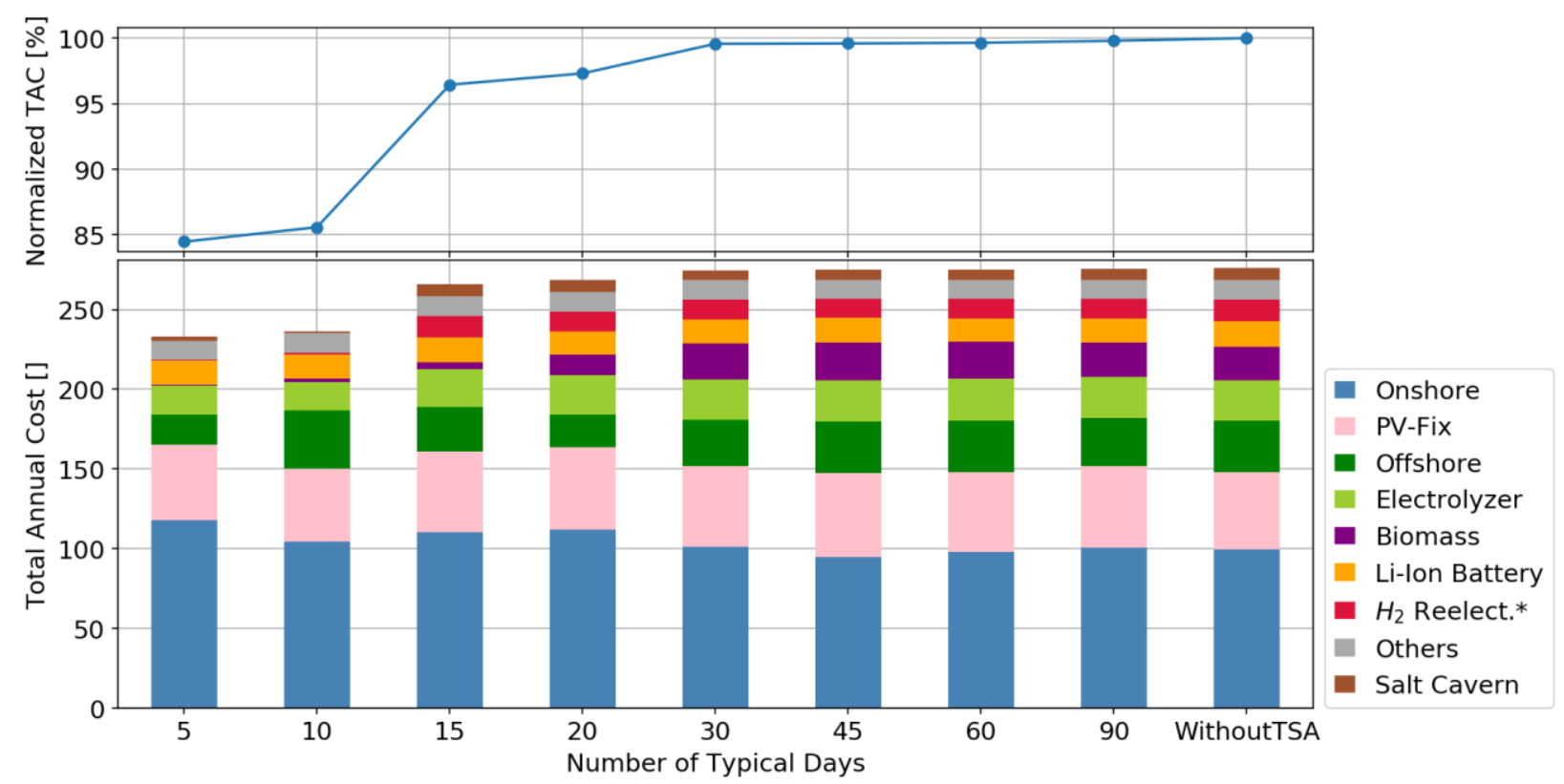

Figure 6. Variation in the TAC with respect to the number of typical days.

Drawing conclusions based on the variations in the total annual cost is not sufficient to understand the variations. Although the shares of technologies do not change, their distribution might alter, keeping the total capacities nearly the same. Thus, the distribution of VRES 
technologies and the optimal pipeline connections are shown in Figure 7 for 5, 10 and 30 typical days, respectively, in addition to the results without time series aggregation.

Looking at the regions connected via pipeline and their respective capacities reveals that the design changes significantly, especially for 5 and 10 typical days. These discrepancies are more pronounced in the Nordic countries, the United Kingdom and Ireland. The optimal capacities in these regions are underestimated by nearly half of their optimum capacity in the reference case. Not only the capacity variations, but also the connections in the cases of 5 and 10 typical days apparently differ from those of the reference scenario (especially in northern Norway and Sweden). Furthermore, Spain and Portugal also experience variations in their pipeline connections. For example, regions in Portugal are not connected to the others in the reference scenario; however, the connections in that area alter in all of the other cases when time series aggregation is used. Southern Portugal is connected to northern Portugal and western Spain when 5 typical days are assumed, whereas it is only connected to southwestern Spain in the 10 typical days case.

In addition to the pipeline connections, the optimal capacities of generation technologies vary drastically in these systems. For instance, except in the case of 5 typical days, southern Sweden utilizes offshore wind energy in all the scenarios. Similarly, change in the technologies between onshore and offshore wind energies is observed in many regions such as northwestern Germany, northeastern Poland and northwestern Spain. The main reason for this switch is the lack of representation of time series for these technologies due to fewer typical days, as nearly 1000 individual time series consisting of 8760 time steps are represented by using 5 normalized daily profiles scaled to match the total generation-consumption. Therefore, considering the different daily profile patterns of hydropower inflow time series, PV and wind technologies, a clear distinction and accurate representation are not possible. As a result, the accuracy of the prediction increases with more typical days with an exponential increase in the solution time.

Overall, hourly time series of this system for the year 2015 can be represented by 30 typical days with slight variations in the resulting system design. However, it must be noted that the variation in the weather time series might alter the number of typical days that can represent these time series. As is indicated by Caglayan et al. [25], selection of the weather year plays a significant role in energy system design. Variation in the generation time series of VRES technologies are a strong function of the weather patterns, which alter from year to year. However, within the scope of this work, the analysis of individual weather years is not possible mainly because of the requisite computational power intensity. Nevertheless, the variation caused by different weather years is expected as another source for uncertainty besides time series aggregation. 

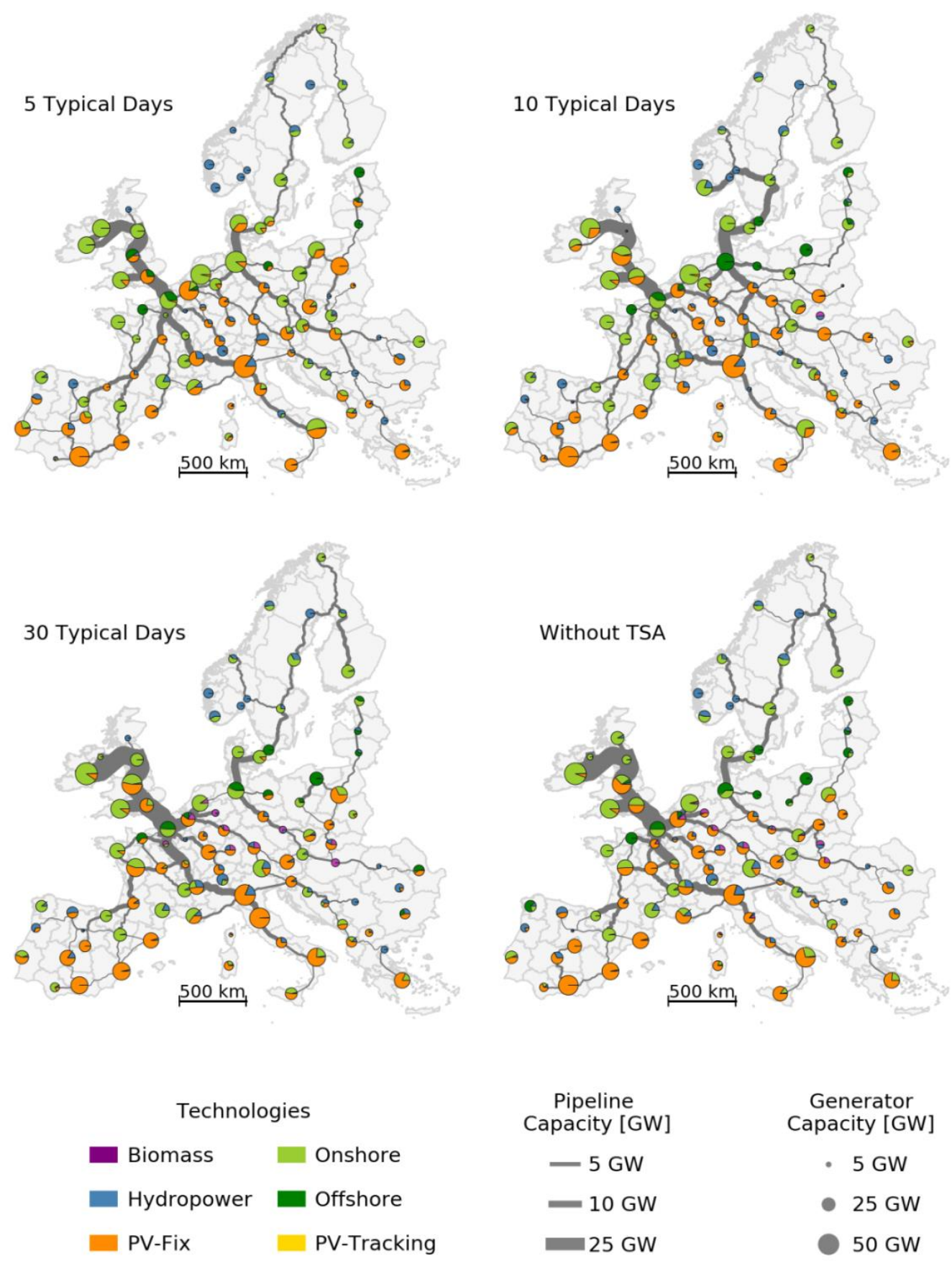

$$
\begin{gathered}
\text { Generator } \\
\text { Capacity [GW] } \\
\text {. } 5 \mathrm{GW} \\
25 \mathrm{GW} \\
50 \mathrm{GW}
\end{gathered}
$$

Figure 7. Capacity distributions of generation technologies and hydrogen pipelines for different numbers of typical days $(5,10$ and 30$)$ and without time series aggregation.

\section{SUMMARY AND OUTLOOK}

An analysis of a $100 \%$ renewable European energy system was conducted that assumed the introduction of hydrogen into the system. Unlike existing studies, high spatial and temporal resolutions were employed (hourly time series across 96 European regions). Moreover, consideration of hydrogen transmission and seasonal storage enhance the uniqueness of this 
work. The optimization of the energy system without time series aggregation results in a generation technology mix as follows: $737 \mathrm{GW}$ onshore, $125 \mathrm{GW}$ offshore, $844 \mathrm{GW}$ open-field PV without tracking and $33 \mathrm{GW}$ biomass CHP plants. Due to the higher investment costs, openfield PV with tracking and rooftop PV are not installed in any regions. Similarly, PEM fuel cells, solid oxide fuel cells and hydrogen gas engines are not preferred in the system, either. A total deployment of $187 \mathrm{TWh}$ of salt caverns, $1 \mathrm{TWh}$ of lithium-ion battery and $0.7 \mathrm{TWh}$ of vessels is observed.

The application of time series aggregation in a European context for many typical periods was conducted. According to the results, misrepresentation of the overall time series is apparent when few typical periods are used (i.e., up to 30 typical days). Due to the lack of representation in the time series, storage and transmission capacities in particular are underestimated. Nevertheless, in spite of few changes, the system design does not significantly alter after 30 typical days.

In this analysis, the problem is simplified by using the average time series of all possible placements within a region. Nevertheless, higher fidelity and spatial resolution can be attained by consideration of different classes for these technologies. The analysis is only conducted for the weather year 2015, which has the inflow time series for hydropower plants. Consideration of different weather years to ensure the security of supply will therefore be necessary for future energy system designs.

\section{ACKNOWLEDGMENTS}

This work was supported by the Helmholtz Association under the Joint Initiative, "EnergySystem 2050: A Contribution of the Research Field Energy". In addition, the authors acknowledge financial support by the Federal Ministry for Economic Affairs and Energy of Germany in the context of project METIS (project number 03ET4064A).

\section{REFERENCES}

[1] Olivier JGJ, Peters JAH. Trends in global CO2 and total greenhouse gas emissions: 2018 Report. The Hague: 2018.

[2] IRENA. Renewable Capacity Statistics 2019. 2019.

[3] Albadi MH, El-Saadany EF. Overview of wind power intermittency impacts on power systems. Electr Power Syst Res 2010;80:627-32.

[4] Schiebahn S, Grube T, Robinius M, Tietze V, Kumar B, Stolten D. Power to gas: Technological overview, systems analysis and economic assessment for a case study in Germany. Int J Hydrogen Energy 2015;40:4285-94. doi:10.1016/j.ijhydene.2015.01.123.

[5] IRENA. Hydrogen from renewable energy: Technology outlook for the energy transition. Abu Dhabi: 2018.

[6] Robinius M, Otto A, Heuser P, Welder L, Syranidis K, Ryberg D, et al. Linking the Power and Transport Sectors-Part 1: The Principle of Sector Coupling. Energies 2017;10:956. doi:10.3390/en10070956.

[7] Aboumahboub T, Tzscheutschler P, Hamacher T. Optimizing World-wide Utilization of Renewable Energy Sources in the Power Sector. Renew Energy Power Qual J 2010;1:1521. doi:10.24084/repqj08.207.

[8] Siala K, Mahfouz MY. Impact of the choice of regions on energy system models. Energy 
Strateg Rev 2019;25:75-85. doi:10.1016/j.esr.2019.100362.

[9] Zappa W, Junginger M, van den Broek M. Is a 100\% renewable European power system feasible by 2050? Appl Energy 2019;233-234:1027-50. doi:10.1016/j.apenergy.2018.08.109.

[10] Bussar C, Moos M, Alvarez R, Wolf P, Thien T, Chen H, et al. Optimal Allocation and Capacity of Energy Storage Systems in a Future European Power System with 100\% Renewable Energy Generation. Energy Procedia 2014;46:40-7. doi:10.1016/j.egypro.2014.01.156.

[11] Bussar C, Stöcker P, Cai Z, Moraes Jr. L, Magnor D, Wiernes P, et al. Large-scale integration of renewable energies and impact on storage demand in a European renewable power system of 2050-Sensitivity study. J Energy Storage 2016;6:1-10. doi:10.1016/j.est.2016.02.004.

[12] Bussar C, Stöcker P, Cai Z, Moraes L, Alvarez R, Chen H, et al. Large-scale Integration of Renewable Energies and Impact on Storage Demand in a European Renewable Power System of 2050. Energy Procedia 2015;73:145-53. doi:10.1016/j.egypro.2015.07.662.

[13] Steinke F, Wolfrum P, Hoffmann C. Grid vs. storage in a 100\% renewable Europe. Renew Energy 2013;50:826-32. doi:10.1016/j.renene.2012.07.044.

[14] Schlott M, Kies A, Brown T, Schramm S, Greiner M. The impact of climate change on a cost-optimal highly renewable European electricity network. Appl Energy 2018;230:1645-59. doi:10.1016/j.apenergy.2018.09.084.

[15] Welder L, Ryberg DS, Kotzur L, Grube T, Robinius M, Stolten D. Spatio-temporal optimization of a future energy system for power-to-hydrogen applications in Germany. Energy 2018;158:1130-49. doi:10.1016/j.energy.2018.05.059.

[16] Samsatli S, Staffell I, Samsatli NJ. Optimal design and operation of integrated windhydrogen-electricity networks for decarbonising the domestic transport sector in Great Britain. Int J Hydrogen Energy 2016;41:447-75. doi:10.1016/j.ijhydene.2015.10.032.

[17] Robinius M, Otto A, Syranidis K, Ryberg DS, Heuser P, Welder L, et al. Linking the Power and Transport Sectors-Part 2: Modelling a Sector Coupling Scenario for Germany. Energies 2017;10:957. doi:10.3390/en10070957.

[18] Hörsch J, Hofmann F, Schlachtberger D, Brown T. PyPSA-Eur: An open optimisation model of the European transmission system. Energy Strateg Rev 2018;22:207-15. doi:10.1016/j.esr.2018.08.012.

[19] Crotogino F, Donadei S, Bünger U, Landinger H, Crotogino F, Donadei S, et al. LargeScale Hydrogen Underground Storage for Securing Future Energy Supplies Large-Scale Hydrogen Underground Storage for Securing Future Energy Supplies. Proc. hte 18th World Hydrog. Energy Conf. 2010, May 16-21 2010, Ess., vol. 78-4, 2010.

[20] Ryberg DS, Caglayan DG, Schmitt S, Linßen J, Stolten D, Robinius M. The future of European onshore wind energy potential: Detailed distribution and simulation of advanced turbine designs. Energy 2019. doi:10.1016/J.ENERGY.2019.06.052.

[21] Caglayan DG, Ryberg DS, Heinrichs H, Linßen J, Stolten D, Robinius M. The technoeconomic potential of offshore wind energy with optimized future turbine designs in Europe. Appl Energy 2019;255:113794. doi:10.1016/j.apenergy.2019.113794.

[22] Caglayan DG, Weber N, Heinrichs H, Linßen J, Robinius M, Kukla PA, et al. Theoratical Potential of Salt Caverns for Hydrogen Storage. 2018.

[23] Welder L, Heinrichs H, Linßen J, Robinius M, Stolten D. Framework for Integrated Energy Systems Assessment (FINE) 2018. https://github.com/FZJ-IEK3-VSA/FINE. 
[24] Bruninx K, Orlic D, Couckuyt D, Grisey N, Betraoui B, Anderski T, et al. Modular Development Plan of the Pan-European Transmission System 2050 - D2.1: Data sets of scenarios for 2050. 2015.

[25] Caglayan DG, Heinrichs HU, Linssen J, Robinius M, Stolten D. Impact of different weather years on the design of hydrogen supply pathways for transport needs. Int $\mathbf{J}$ Hydrogen Energy 2019;44:25442-56. doi:10.1016/J.IJHYDENE.2019.08.032.

[26] Rienecker MM, Suarez MJ, Gelaro R, Todling R, Bacmeister J, Liu E, et al. MERRA: NASA's Modern-Era Retrospective Analysis for Research and Applications. J Clim 2011;24:3624-48. doi:10.1175/JCLI-D-11-00015.1.

[27] Syranidis K, Markowitz P, Linssen J, Robinius M, Stoltcn D. Flexible Demand for Higher Integration of Renewables into the European Power System. 2018 15th Int. Conf. Eur. Energy Mark., IEEE; 2018, p. 1-6. doi:10.1109/EEM.2018.8469962.

[28] J. Carlsson, M. del Mar Perez Fortes, G. de Marco, J. Giuntoli, M. Jakubcionis, A. JägerWaldau, R. Lacal-Arantegui S, Lazarou, D. Magagna, C. Moles, B. Sigfusson, A. Spisto, M. Vallei, EW. ETRI 2014 - Energy Technology Reference Indicator projections for 2010-2050. 2014. doi:10.2790/057687.

[29] Ryberg DS, Caglayan DG, Schmitt S, Linßen J, Robinius M, Stolten D. Variable-Capacity Cost Analysis of Wind Production via the Distribution and Simulation of Single Turbines. 2018.

[30] Directorate-General for Energy (European Commission). METIS Studies - The role and potential of Power-to-X in 2050. 2019. doi:10.2833/459958.

[31] Dincer I, Acar C. Review and evaluation of hydrogen production methods for better sustainability. Int J Hydrogen Energy 2014. doi:10.1016/j.ijhydene.2014.12.035.

[32] Welder L, Stenzel P, Ebersbach N, Markewitz P, Robinius M, Emonts B, et al. Design and evaluation of hydrogen electricity reconversion pathways in national energy systems using spatially and temporally resolved energy system optimization. Int $\mathbf{J}$ Hydrogen Energy 2019;44:9594-607. doi:10.1016/j.ijhydene.2018.11.194.

[33] Schlachtberger DP, Brown T, Schramm S, Greiner M. The benefits of cooperation in a highly renewable European electricity network. Energy 2017;134:469-81. doi:10.1016/J.ENERGY.2017.06.004.

[34] ENTSO-E. 10-Year Network Development Plan 2012. 2012.

[35] Reuß M, Grube T, Robinius M, Preuster P, Wasserscheid P, Stolten D. Seasonal storage and alternative carriers: A flexible hydrogen supply chain model. Appl Energy 2017;200:290-302. doi:10.1016/j.apenergy.2017.05.050.

[36] WorldMap. Natural Gas Pipelines in Europe, Asia, Africa \& Middle East n.d. https://worldmap.harvard.edu/data/geonode:natural_gas_pipelines_j96 (accessed April 4, 2017).

[37] Ryberg DS, Tulemat Z, Robinius M, Stolten D. Geospatial Land Availability for Energy Systems (GLAES) 2017. 